\title{
RECURSIVE FORMULAE FOR THE MULTIPLICATIVE PARTITION FUNCTION
}

\author{
JUN KYO KIM and SANG GUEN HAHN
}

(Received 28 February 1996)

\begin{abstract}
For a positive integer $n$, let $f(n)$ be the number of essentially different ways of writing $n$ as a product of factors greater than 1, where two factorizations of a positive integer are said to be essentially the same if they differ only in the order of the factors. This paper gives a recursive formula for the multiplicative partition function $f(n)$.
\end{abstract}

Keywords and phrases. Partitions, multiplicative partitions.

1991 Mathematics Subject Classification. 11P82.

A multi-partite number of order $j$ is a $j$-dimensional vector, the components of which are nonnegative integers. A partition of $\left(n_{1}, n_{2}, \ldots, n_{j}\right)$ is a solution of the vector equation

$$
\sum_{k}\left(n_{1 k}, n_{2 k}, \ldots, n_{j k}\right)=\left(n_{1}, n_{2}, \ldots, n_{j}\right)
$$

in multi-partition numbers other than $(0,0, \ldots, 0)$. Two partitions which differ only in the order of the multi-partite numbers are regarded as identical. We denote by $p\left(n_{1}, n_{2}, \ldots, n_{j}\right)$ the number of different partitions of $\left(n_{1}, n_{2}, \ldots, n_{j}\right)$. For example, $p(3)=3$ since $3=2+1=1+1+1$ and $p(2,1)=4$ since $(2,1)=(2,0)+(0,1)=$ $(1,0)+(1,0)+(1,0)=(1,0)+(1,1)$. Let $f(1)=1$ and for any integer $n>1$, let $f(n)$ be the number of essentially different ways of writing $n$ as a product of factors greater than 1 , where two factorizations of a positive integer are said to be essentially the same if they differ only in the order of the factors. For example, $f(12) p(2,1)=4$ since $12=$ $2 \cdot 6=3 \cdot 4=2 \cdot 2 \cdot 3$. In general, if $n=p_{1}^{n_{1}} p_{2}^{n_{2}} \cdots p_{j}^{n_{j}}$, then $f(n)=p\left(n_{1}, n_{2}, \ldots, n_{j}\right)$. We find recursive formulas for the multi-partite partition function $p\left(n_{1}, n_{2}, \ldots, n_{j}\right)$. The most useful formula known to this day for actual evaluation of the multi-partite partition function is presented in Theorem 4.

For convenience, we define some sets used in this paper. For a positive integer $r$, let $M_{r}^{0}$ be the set of $r$-dimensional vectors with nonnegative integer components and $M_{r}$ be the set of $r$-dimensional vectors with nonnegative integer components not all of which are zero. The following three theorems are well known.

THEOREM 1 (Euler [3]; see also [1, p. 2]). If $n \geq 0$, then

$$
p(n)=\sum_{m=1}^{\infty}(-1)^{m+1}\left(p\left(n-\frac{1}{2} m(3 m-1)\right)+p\left(n-\frac{1}{2}(3 m+1)\right)\right),
$$

where we recall that $p(k)=0$ for all negative integers $k$. 
THEOREM 2. If $n \geq 0$, then $p(0)=1$ and

$$
n \cdot p(n)=\sum_{k=1}^{n} \sigma(k) \cdot p(n-k),
$$

where $\sigma(m)=\sum_{d \mid m} d$.

THEOREM 3 ([1, Ch. 12]). If $g\left(x_{1}, x_{2}, \ldots, x_{r}\right)$ is the generating function for $p(\vec{n})$ and $\left|x_{i}\right|<1$ for $i \leq r$, then

$$
\begin{aligned}
g\left(x_{1}, x_{2}, \ldots, x_{r}\right) & =\prod_{\vec{n} \in M_{r}} \frac{1}{1-x_{1}^{n_{1}} x_{2}^{n_{2}} \cdots x_{r}^{n_{r}}} \\
& =1+\sum_{\vec{m} \in M_{r}} p(\vec{m}) x_{1}^{m_{1}} x_{2}^{m_{2}} \cdots x_{r}^{m_{r}} .
\end{aligned}
$$

Similarly, we can extend the equation of Theorem 2 to multi-partite numbers as follows.

THEOREM 4. For $\vec{n} \in M_{r}$, we have

$$
n_{i} \cdot p(\vec{n})=\sum_{\substack{l_{j} \leq n_{j} \text { for } \\ \vec{l} \in M_{r}}} \frac{\sigma(\operatorname{gcd}[\vec{l}])}{\operatorname{gcd}[\vec{l}]} \cdot l_{i} \cdot p(\vec{n}-\vec{l})
$$

Proof. Let $g\left(x_{1}, x_{2}, \ldots, x_{r}\right)$ be the function defined in Theorem 3. Taking the $i$ th partial logarithmic derivative of the product formula for $g\left(x_{1}, x_{2}, \ldots, x_{r}\right)$ in (4), we get

$$
\begin{aligned}
\frac{\partial g\left(x_{1}, x_{2}, \ldots, x_{r}\right)}{\partial x_{i}} \cdot \frac{x_{i}}{g\left(x_{1}, x_{2}, \ldots, x_{r}\right)} & =\sum_{i \in M_{r}} \frac{l_{i} \cdot \prod_{j=1}^{r} x_{j}^{l_{j}}}{1-\prod_{j=1}^{r} x_{j}^{l_{j}}} \\
& =\sum_{i \in M_{r}} \sum_{k=1}^{\infty} l_{i} \cdot\left(\prod_{j=1}^{r} x_{j}^{l_{j}}\right)^{k} .
\end{aligned}
$$

Taking the $i$ th partial derivative of the right-hand side of (4), we get

$$
\begin{aligned}
\sum_{\vec{n} \in M_{r}} n_{i} \cdot p(\vec{n}) x_{1}^{n_{1}} x_{2}^{n_{2}} \cdot x_{r}^{n_{r}} & =\frac{\partial g\left(x_{1}, x_{2}, \ldots, x_{r}\right)}{\partial x_{i}} \cdot x_{i} \\
& =g\left(x_{1}, x_{2}, \ldots, x_{r}\right) \sum_{\vec{t} \in M_{r}} \sum_{k=1}^{\infty} t_{i} \cdot\left(\prod_{j=1}^{r} x_{j}^{t_{j}}\right)^{k} \\
& =\left(\sum_{\vec{m} \in M_{r}^{0}} p(\vec{m}) x_{1}^{m_{1}} x_{2}^{m_{2}} \cdots x_{r}^{m_{r}}\right) \sum_{\vec{t} \in M_{r}} \sum_{k=1}^{\infty} t_{i} \cdot\left(\prod_{j=1}^{r} x_{j}^{t_{j}}\right)^{k} .
\end{aligned}
$$

Comparing the coefficients of both sides of (7), we get 


$$
\begin{aligned}
n_{i} \cdot p(\vec{n}) & =\sum_{\substack{\vec{m}, \vec{t} \in M_{r}^{0}, k \in M_{1} \\
\vec{m}+k \vec{t}=\vec{n}}} t_{i} \cdot p(\vec{m}) \\
& =\sum_{\vec{l} \in M_{r}} p(\vec{n}-\vec{l}) \sum_{k \mid \operatorname{gcd}(\vec{l})} \frac{l_{i}}{k} \\
& =\sum_{\substack{l_{j} \leq n_{j} \text { for } j \leq r \\
\vec{l} \in M_{r}}} \frac{\sigma(\operatorname{gcd}[\vec{l}])}{\operatorname{gcd}[\vec{l}]} \cdot l_{i} \cdot p(\vec{n}-\vec{l}) .
\end{aligned}
$$

The theorem is proved.

COROLlary 5. For $\vec{n} \in M_{r}$, we have

$$
\left(\sum_{i=1}^{r} n_{i}\right) \cdot p(\vec{n})=\sum_{\substack{l_{j} \leq n_{j} \text { for } j \leq r \\ \vec{l} \in M_{r}}} \frac{\sigma(\operatorname{gcd}[\vec{l}])}{\operatorname{gcd}[\vec{l}]}\left(\sum_{i=1}^{r} l_{i}\right) \cdot p(\vec{n}-\vec{l}) .
$$

For positive integers $m$ and $n$, let

$$
(m, n)_{\models}=\max _{\substack{k \mid m \\ n^{1 / k} \text { is an integer }}} k .
$$

The following properties of $(m, n)_{\vDash}$ are easy to obtain:

(1) $\left(m, p_{1}^{n_{1}} p_{2}^{n_{2}} \cdot p_{k}^{n_{k}}\right)=\operatorname{gcd}\left(m, n_{1}, n_{2}, \ldots, n_{k}\right)$

(2) $(m, n k)_{\models}=\operatorname{gcd}\left[(m, n)_{\models},(m k)_{\models}\right]$ for $\operatorname{gcd}(n, k)=1$

(3) $(m k, n)_{\vDash}=(m, n)_{\vDash} \cdot(k, n)_{\vDash}$ for $\operatorname{gcd}(m, k)=1$.

From the point of view of the multiplicative partition function, Theorem 4 can be restated as the following theorem.

THEOREM 6. let $n, t$ be positive integers and let $p$ be a prime number such that $p \nmid m$. Then we get

$$
t \cdot f\left(m p^{t}\right)=\sum_{i=1}^{t} \sum_{l \mid m} \frac{\sigma\left((i, l)_{\models}\right)}{(i, l)_{\models}} i \cdot f\left(\frac{m}{l} p^{t-i}\right) .
$$

In [4], MacMahon presents a table of values of $f(n)$ for those $n$ which divide one of $2^{10} \cdot 3^{8}, 2^{10} \cdot 3 \cdot 5,2^{9} \cdot 3^{2} \cdot 5^{1}, 2^{8} \cdot 3^{3} \cdot 5^{1}, 2^{6} \cdot 3^{2} \cdot 5^{2}, 2^{5} \cdot 3^{3} \cdot 5^{2}$. In [2], Canfield, Erdös, and Pomerance commented that they doubted the correctness of MacMahon's figures. Specifically,

$$
\begin{aligned}
p(10,5) & =3804, & & \operatorname{not} 3737, \\
p(9,8) & =13715, & & \text { not } 13748, \\
p(10,8) & =21893, & & \text { not } 21938, \\
p(4,1,1) & =38, & & \operatorname{not} 28 .
\end{aligned}
$$

From Theorem 4 we can easily be sure that Canfield, Erdös and Pomerance comment is true. 


\section{REFERENCES}

[1] G. E. Andrews, The theory of partitions, Encyclopedia of Mathematics and its Applications, vol. 2, Addison-Wesley Publishing Co., London,Amsterdam, 1976. MR 5827738. Zbl 371.10001.

[2] E. R. Canfield, Paul Erdos, and Carl Pomerance, On a problem of Oppenheim concerning "factorisatio numerorum", J. Number Theory 17 (1983), no. 1, 1-28. MR 85j:11012. Zbl 513.10043.

[3] L. Euler, Introductio in Analysin Infinitorum, ch. 16, Marcum-Michaelem Bousquet Lausannae, 1748 (Latin).

[4] P. A. Macmahon, The enumeration of the partitions of multipartite numbers, Math. Proc. Cambridge Philos. Soc. 22 (1927), 951-963.

Kim AND HAHN: Korea AdVANCED INSTITUTE OF SCIENCE AND TeCHNOLOGy 373-1, Kusong Don, Yusung Gu, TAEJON 305-701, SOUTH Korea 


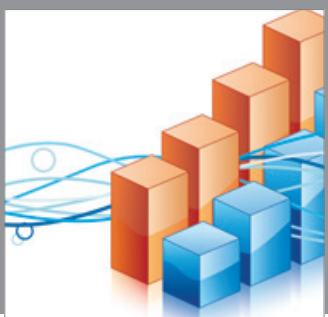

Advances in

Operations Research

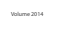

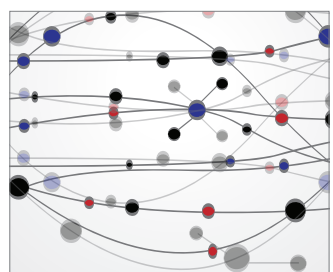

\section{The Scientific} World Journal
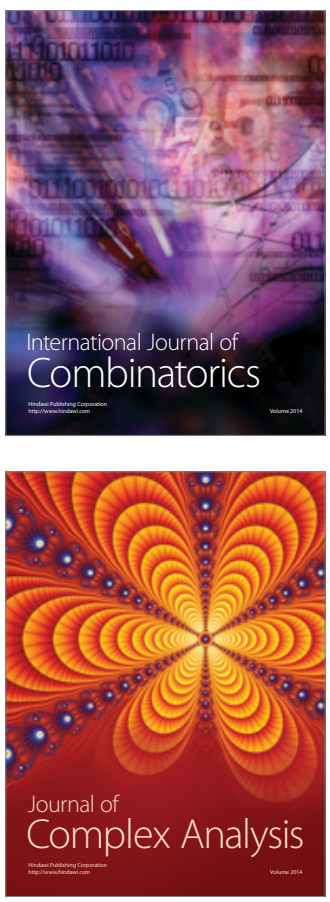

International Journal of

Mathematics and

Mathematical

Sciences
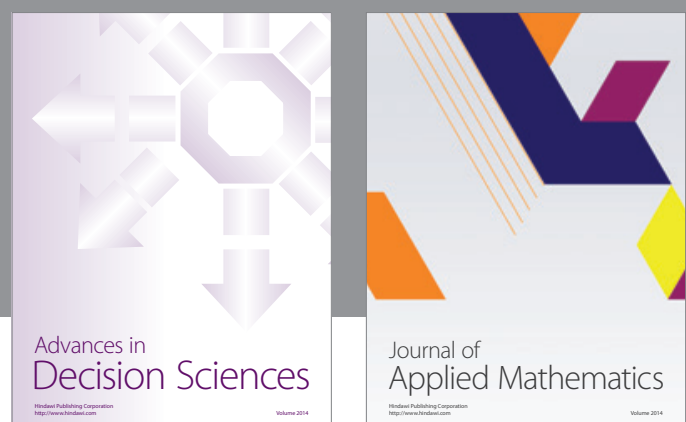

Journal of

Applied Mathematics
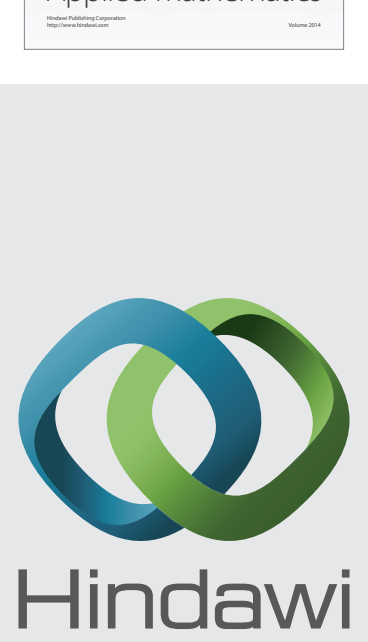

Submit your manuscripts at http://www.hindawi.com
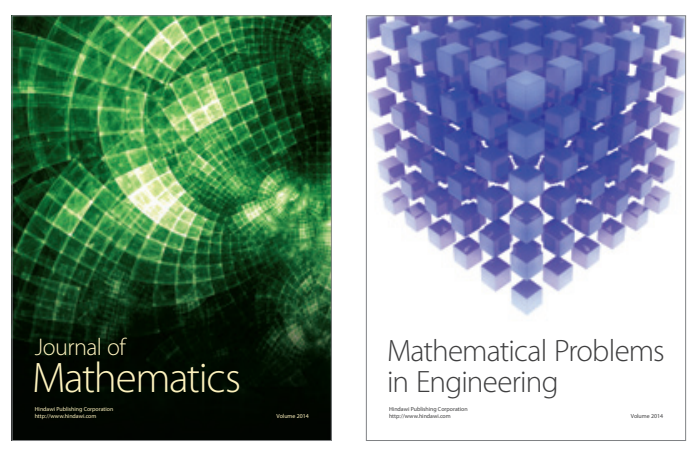

Mathematical Problems in Engineering
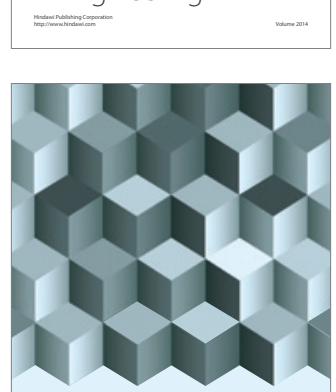

Journal of

Function Spaces
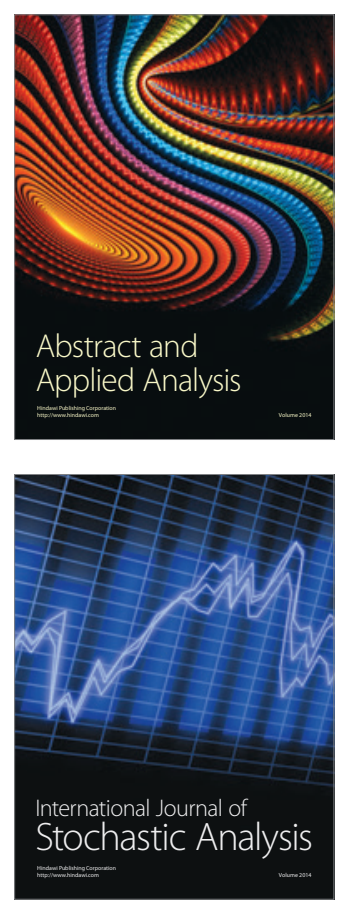

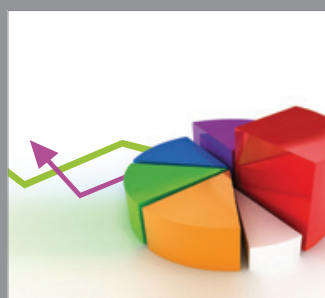

ournal of

Probability and Statistics

Promensencen
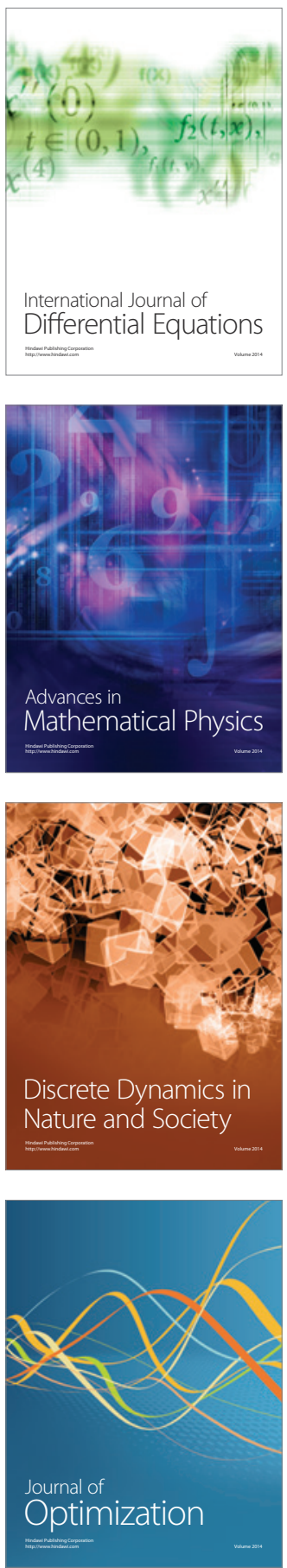